msh-mss Mathématiques et sciences humaines

183 | Automne 2008

Hommage à Georges-Th. Guilbaud

\title{
G.-Th. Guilbaud
}

G.-Th. Guilbaud

Marc Barbut

\section{(2) OpenEdition}

Journals

Édition électronique

URL : http://journals.openedition.org/msh/10733

DOI : $10.4000 / \mathrm{msh} .10733$

ISSN : 1950-6821

\section{Éditeur}

Centre d'analyse et de mathématique sociales de l'EHESS

\section{Édition imprimée}

Date de publication : 14 décembre 2008

ISSN : 0987-6936

\section{Référence électronique}

Marc Barbut, «G.-Th. Guilbaud », Mathématiques et sciences humaines [En ligne], 183 | Automne 2008, mis en ligne le 15 décembre 2008, consulté le 23 juillet 2020. URL : http://journals.openedition.org/ msh/10733; DOI : https://doi.org/10.4000/msh.10733 
G.-Th. GUILBAUD (1912-2008)

Georges Th. Guilbaud, mort le dimanche de Pâques à 95 ans, a joué un rôle considérable pour la diffusion des techniques et des idées mathématiques, dans le monde de l'entreprise, des administrations et des économistes d'une part, dans celui de la recherche en sciences humaines et sociales d'autre part.

Sa prodigieuse activité de chercheur, d'enseignant et d'agitateur - il qualifiait souvent d' « agit-prop » ce qu'il a toujours considéré comme un apostolat - s'est exercée dans trois grands domaines scientifiques :

- Recherche opérationnelle, mathématiques de la décision et calcul économique.

- Mathématiques sociales (au sens de Condorcet) appliquées notamment aux théories du choix social, à celles des systèmes de parenté, de la perception de l'espace ou à la lexicométrie...

- Histoire et épistémologie des mathématiques, et singulièrement du calcul des probabilités et de la statistique.

Sur le premier point, nul de ceux qui y ont participé n'a oublié l'enthousiasme des ingénieurs et élèves des Écoles qui se pressaient, au tournant des années 50-60, à son séminaire de recherche opérationnelle de l'Institut Henri Poincaré.

Guilbaud fut en effet, dans l'immédiat après-guerre, et alors qu'il était, de 1947 à 1955 membre puis directeur adjoint de l'Institut de Sciences Économiques Appliquées (ISEA) de François Perroux, celui qui introduisit en France la toute nouvelle théorie des jeux de von Neumann et Morgenstern, et les techniques de recherche opérationnelle élaborées dans les pays anglo-saxons pour faire face aux nécessités de l'économie de guerre. C'est lui qui créa et dirigea le Bureau universitaire de recherche opérationnelle de la Faculté des sciences de Paris, et fut en 1956 fondateur et premier président de la Société Française de Recherche Opérationnelle (SOFRO).

Lorsque l'on décida, à la fin des années 50 de réformer les études d'économie politique et d'y inclure un enseignement de mathématiques au programme ambitieux, c'est à Guilbaud que fut confiée l'organisation de cet enseignement à la Faculté de Droit et Sciences Économiques de Paris ; à cet effet, il y fut nommé temporairement professeur de 1959 à 1963.

En 1955, Guilbaud est élu directeur d'études, sous l'intitulé «Méthodes mathématiques des sciences sociales », à la $6^{\mathrm{e}}$ section de l'EPHE, où Lucien Fèbvre et Charles Morazé l'ont appelé.

C'est donc dans le cadre de notre École, qu'il ne quitta plus jusqu'à sa retraite en 1981, qu'il exerce désormais ses activités, abandonnant progressivement le volet «calcul économique » au profit de ceux des «mathématiques sociales » et de l'histoire des idées mathématiques. 
Il crée la revue Mathématiques et Sciences humaines (1962) et le Centre de mathématique sociale, devenu en 1981 Centre d'Analyse et de Mathématique Sociales (CAMS).

Les fidèles de ses séminaires de l'École, ce sont encore quelques ingénieurs, mais surtout des psychologues, anthropologues, sociologues, linguistes ou philosophes ; voire même quelques historiens ; et des mathématiciens.

Dans ces « années 60 », de très fructueuses collaborations se nouent avec d'autres centres de recherche de notre École, au premier rang desquels le Laboratoire de psychologie génétique de François Bresson et Pierre Greco, et le Laboratoire d'anthropologie sociale de Claude Levi-Strauss ; mais bien d'autres encore, dans l'École et dans les Facultés, voire même en dehors de toute institution universitaire, comme avec le compositeur Iannis Xenakis, dont l'Equipe de Mathématique Musicale $(\mathrm{EMaMu})$ fut créée dans le Centre de mathématique sociale.

Et, bien sûr, lors de la réforme de 1966 des cursus de sciences humaines (réforme Fouchet) qui introduit des mathématiques dans les études de psychologie, de sociologie et de philosophie, c'est l'équipe que Guilbaud a réunie autour de lui au Centre de mathématique sociale ${ }^{1}$ qui est chargée de la définition des programmes et de l'organisation des enseignements dans les Facultés des lettres et sciences humaines de Paris-Sorbonne et de Paris-Nanterre. D'ailleurs, lui-même y vécut intensément, c'était dans son tempérament, les «événements » du printemps 1968.

L'intérêt de Guilbaud pour les mathématiques dans les sciences sociales est inséparable de celui qu'il a toujours eu pour l'histoire des mathématiques : quel meilleur moyen de faire comprendre de l'intérieur une idée mathématique que d'en exposer la genèse, textes originaux à l'appui ?

Car G.-Th. Guilbaud, ce mathématicien, fut aussi un grand érudit, un humaniste chrétien imprégné de l'oeuvre de Blaise Pascal. C'est notamment grâce à lui que dès les années 40 (lorsque de 1942 à 1946, il était professeur de mathématiques spéciales au Lycée de Dijon où G. G. Granger enseignait la philosophie) les travaux de Condorcet en « mathématique sociale » furent tirés de l'oubli.

Depuis sa création en 1982-1983, jusqu'au moment où il se consacra essentiellement à sa femme atteinte d'une longue et douloureuse maladie, Guilbaud fut l'un des principaux animateurs du séminaire d'Histoire du calcul des probabilités et de la statistique.

G.-Th. Guilbaud laisse derrière lui une oeuvre considérable : 11 livres, plus de 100 articles dont beaucoup ont fait date, et certains ont été traduits dans diverses revues scientifiques étrangères.

Ancien élève de l'ENS-Ulm (1932-1935), agrégé de mathématiques, il a obtenu dans sa discipline des résultats importants (en théorie du vote et en combinatoire notamment) qu'il n'a jamais voulu affubler du nom, qu'il jugeait un peu pompeux, de « théorème », et qu'il a toujours négligé - il l'a parfois regretté - de faire « breveter ». Il disait fréquemment : «On a l'âge de ses algèbres ». À cet égard, il est sûr qu'il resta un éternel jeune homme.

Il eut aussi un extraordinaire talent pour enseigner et faire aimer les mathématiques, sur lesquelles un autre de ses dictons favoris était: "Les mathématiques, c'est comme le cochon. Tout y est bon ». Il est vrai qu'il avait fait son

\footnotetext{
${ }^{1}$ Micheline Petruszewycz, Marc Barbut, Charlotte Carcassonnet, Michel Eytan, Pierre Rosenstiehl, Bernard Monjardet, Bruno Leclerc, Claude Le Conte de Poly.
} 
apprentissage à la meilleure école, celle de l'enseignement secondaire (il fut professeur de lycée de 1936 à 1946). Aucun de ceux qui ont assisté à ses cours et séminaires n'oubliera sa manière profondément originale de faire comprendre des mathématiques à des publics de non spécialistes, la clarté de ses exposés, son enthousiasme pour sa discipline et sa foi en les vertus de celle-ci. Il en fut un incomparable « passeur ».

Sur ce point, et en conclusion, laissons témoigner l'un de ceux qui firent partie de ces publics, le psycho-sociologue Claude Flament ${ }^{2}$ : «Guilbaud n'aimait pas la psychologie; je l'ai entendu la qualifier de science cruelle, et c'était à peine une plaisanterie ! La question n'est pas là : je veux me souvenir de sa manière de nous enseigner l'esprit mathématique à travers des formulations (ce n'était pas toujours des formalisations) d'exemples empruntés aux diverses sciences sociales (parfois, même à la psychologie)... Guilbaud m'a appris à penser comme un chercheur, au temps où j'apprenais la technique expérimentale dans le Laboratoire de Fraisse, à la Sorbonne... Apprendre à penser d'une certaine manière est essentiel pour un chercheur, et c'est ce que je dois à Guilbaud».

M.B.

\footnotetext{
${ }^{2}$ Professeur émérite à l'Université de Provence, ancien directeur d'études à l'EHESS.
} 